\title{
Ubiquitinated Protein Degradation
}

National Cancer Institute

\section{Source}

National Cancer Institute. Ubiquitinated Protein Degradation. NCI Thesaurus. Code C21243.

Ubiquitinated Protein Degradation involves the regulated covalent conjug ation of one or more ubiquitin residues to one or more sites on a protein that acts as a tag by which the protein-transport machinery ferries the targeted ubiquinated protein to the proteasome for proteolytic destruction. 